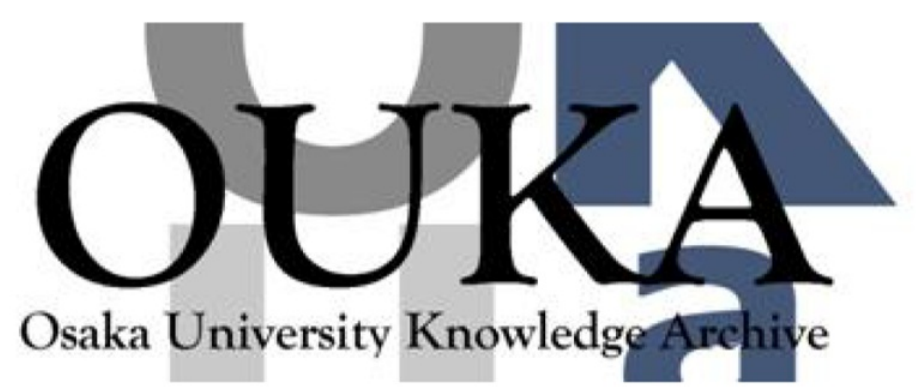

\begin{tabular}{|c|c|}
\hline Title & $\begin{array}{l}\text { Simulations of trench-filling profiles under } \\
\text { ionized magnetron sputter metal deposition }\end{array}$ \\
\hline Author (s) & Hamaguchi, S.; Rossnagel, S. M. \\
\hline Citation & $\begin{array}{l}\text { Journal of Vacuum Science and Technology B: } \\
\text { Microelectronics and Nanometer Structures. } \\
\text { 13(2) p. 183-p. } 191\end{array}$ \\
\hline Issue Date & $1995-03$ \\
\hline oaire:version & VoR \\
\hline URL & https://hdl. handle. net/11094/78503 \\
\hline rights & $\begin{array}{l}\text { This article may be downloaded for personal use } \\
\text { only. Any other use requires prior permission } \\
\text { of the author and AIP Publishing. This article } \\
\text { appeared in Journal of Vacum Science } \& \\
\text { Technology B: Microelectronics and Nanometer } \\
\text { Structures Processing, Measurement, and } \\
\text { Phenomena 13, 183 (1995) and may be found at } \\
\text { https://doi.org/10.1116/1.587995. }\end{array}$ \\
\hline Note & \\
\hline
\end{tabular}

Osaka University Knowledge Archive : OUKA

https://ir. Library. osaka-u. ac. jp/

0saka University 


\title{
Simulations of trench-filling profiles under ionized magnetron sputter metal deposition
}

\author{
S. Hamaguchi and S. M. Rossnagel \\ IBM Thomas J. Watson Research Center; Yorktown Heights. New York 10598
}

(Received 29 June 1994; accepted 28 December 1994)

\begin{abstract}
Numerical simulation results are presented for microscopic profile evolutions of deposited metal films in trench structures under ionized magnetron sputter deposition. The model used for the simulations takes account of the deposition of both ionized and neutral metal species and sputtering (i.e., etching) of the deposited film by the bombardment of metal and inert-gas (such as argon) ions. The evolution of the surface topography is calculated numerically using the shocktracking algorithm. Numerical results are also compared with experimental observations. A primary application of this metal deposition technique is interconnect metallization on semiconductors. (C) 1995 American Vacuum Society.
\end{abstract}

\section{INTRODUCTION}

Recently it has been demonstrated that ionized metal fluxes can be effectively utilized to fill trenches and vias with high aspect ratios (i.e., depth to width ratios) under ionized magnetron sputter deposition. ${ }^{1,2}$ Metal deposition (e.g., Al, $\mathrm{Cu}$. etc.) into such small structures is used for high-density interconnections on semiconductor chips. Other metallization techniques that rely on metal ions include, for example, metal-vapor plasma deposition by electron-cyclotron resonance (ECR)..$^{3-5}$

In typical ionized magnetron sputter deposition, ion fluxes are almost unidirectional (perpendicular to the wafer surface). whereas neutral atom fluxes characteristic of conventional magnetron sputter deposition typically have large angular distributions. The delta-function-like angular distribution of the ion fluxes at the surface is, of course, due to ion acceleration in the sheath region adjacent the wafer. The amount of metal atoms resputtered from the surface by the ion bombardment may be easily controlled by the dc-bias voltage applied to the wafer. With a sufficiently small dc-bias voltage (e.g., $\$ 20 \mathrm{~V}$ ), one may assume that the ion fluxes are practically unidirectional and the sputtering yield is nevertheless negligibly small. With a higher voltage, on the other hand. the deposited metal film becomes subject to etching (i.e.. sputtering) as well as redeposition, which may be used to control the overall filling profiles.

The ratio of the ion flux to the neutral flux in ionized magnetron sputter deposition may also be easily controlled by adjusting the power applied to the radio-frequency induction (RFI) coil. The RFI coil is used to generate a plasma of the metal and working gas (e.g., Ar) near the wafer. The topography of deposited films into microscopic structures is highly dependent on this ratio: higher ion fluxes lead to more anisotropic deposition (due to the directionality of the ion fluxes), whereas higher neutral fluxes generally contribute to the buildup of overhanging metal deposit at the mouth of a structure, often yielding a void inside a deep trench or via.

In this article, we present numerical simulation results for metallization topography in various trench structures. Scanning electron microscope (SEM) pictures taken from ionized magnetron sputter deposition experiments are also presented for comparison with the numerical results. In the simulations, deposition and etch rates are calculated at each point on the surface from the metal-ion, metal-neutral, and inertgas (such as Ar used as a working gas) fluxes incident on the surface. The surface is then advanced in time according to the calculated deposition and etch rates. The numerical method to advance the surface is based on the shock-tracking method. ${ }^{6.7}$ The numerical scheme employed here is sufficiently generic and may be readily applied to other deposition and etch processes such as ECR metal-vapor deposition, plasma-enhanced chemical vapor deposition (PECVD), etc.

The goal of this article is to demonstrate that the model and simulation code provide sufficiently realistic and accurate description of the processes and may be used to facilitate the development of metallization processes. More detailed numerical and experimental data of ionized magnetron sputter deposition will be presented in future publications.

\section{CALCULATIONS OF DEPOSITION AND ETCH RATES}

In this article, we only consider two-dimensional geometrical structures such as infinitely long trenches. Let us take the $x$ axis in the horizontal direction and the $z$ axis in the vertical direction. The $y$ coordinate is chosen accordingly to form the usual right-hand coordinate system. The system is then assumed to have a translational symmetry in the $y$ direction.

Under this coordinate system, the boundary between the solid material and vacuum (or gas phase) at time $t$ may be represented by a curve given by the equation $\psi(x, z, t)=0$. Etching and deposition processes may then be formulated as the time evolution of this surface. If the normal velocity $\mathbf{C}$ of the surface at each point is given, then the equation of motion for the surface is given by

$$
\psi_{1}+C|\nabla \psi|=0
$$

where the direction of the normal velocity $\mathbf{C}$ is taken in such a way that positive $C=|\mathbf{C}|$ indicates the direction of etching. To solve Eq. (1) numerically, we use the shock-tracking method developed in Ref. 6. (In Ref. 6, only cases for etch- 


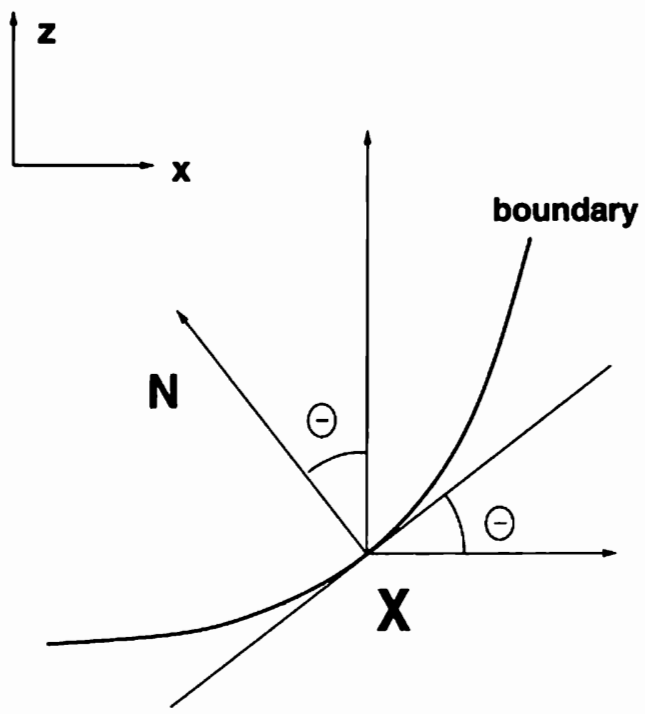

FIG. 1. The curve represents the material boundary.

ing are discussed. It is, however, straightforward to apply the same numerical algorithm to deposition cases simply by reversing the sign of the surface velocity.)

To determine the local surface velocity $\mathbf{C}$, we first calculate how many metal atoms are deposited and reemitted at each point on the surface. For simplicity, we shall assume that the flow and diffusion of the materials along the surface are negligibly small, which is reasonable for most metals on a relatively low-temperature substrate. Although the boundary is essentially two dimensional, the transport in the gas phase is three dimensional since the motions of atoms in the gas phase are not confined in the $x z$ plane.

Suppose the velocity distribution function of species $\alpha$ (e.g., $\mathrm{Al}$ atoms) in the gas phase at $\mathbf{X}$ on the surface is given by $f_{\alpha}(\mathbf{v}, \mathbf{X})$. Then the incoming flux $\mathbf{F}_{\alpha}^{\text {in }}$ at position $\mathbf{X}$ may be calculated as

$$
\mathbf{F}_{\alpha}^{\mathrm{in}}=\int_{\mathbf{N} \cdot \mathbf{v}<0} \mathbf{v} f_{\alpha}(\mathbf{v}, \mathbf{X}) d \mathbf{v},
$$

where $\mathbf{N}$ denotes the unit normal vector of the surface at $\mathbf{X}$ (see Fig. 1). The total number of species $\alpha$ received on the unit area per unit time at $\mathbf{X}$ is then given by $\mathscr{F}_{\alpha}^{\text {in }}=-\mathbf{N} \cdot \mathbf{F}_{\alpha}^{\text {in }}$. (Note that the minus sign arises since $\mathscr{F}_{\alpha}^{\text {in }}>0$.) Likewise, the outgoing flux $\mathbf{F}_{\alpha}^{\text {out }}$-i.e., the flux reemitted from the surface-at $\mathbf{X}$ may be expressed as

$$
\mathbf{F}_{\alpha}^{\text {out }}=\int_{\mathbf{N} \cdot \mathbf{v}>0} \mathbf{v} f_{\alpha}(\mathbf{v}, \mathbf{X}) d \mathbf{v},
$$

and the total number of species $\alpha$ emitted from the unit area per unit time at $\mathbf{X}$ is given by $\mathcal{F}_{\alpha}^{\text {out }}=\mathbf{N} \cdot \mathbf{F}_{\alpha}^{\text {out }}$.

Let us denote the sputtering yields of the metal atoms due to impinging metal atoms of the same kind by $Y_{m}+(\Theta, \varnothing)$ and the sputtering yield due to inert gas ions (such as $\mathrm{Ar}^{+}$) by $Y_{g^{+}}(\Theta, \oslash)$. Here the yields are assumed to depend on the angle $\Theta$ formed by the surface normal and the direction of impinging ions and the ion kinetic energy $\%$. As usual, the sputtering yield is defined as the number of atoms that are removed from the surface due to the collision with a single ion impinging on the surface.

The number of metal atoms that are sputtered from the unit area of the surface per unit time by the metal-ion bombardment is then given by

$$
\int_{\mathbf{N} \cdot \mathbf{v}<0} Y_{m}+(\Theta, \fallingdotseq)(\mathbf{N} \cdot \mathbf{v}) f_{m}+(\mathbf{v}, \mathbf{X}) d \mathbf{v}
$$

where $\mathbf{N} \cdot \mathbf{v}=v \cos \Theta$ and $/=\frac{1}{2} m v^{2}$ with $v=|\mathbf{v}|$ and $m$ being the mass of the incoming metal ion. The velocity distribution function of the incoming metal ions are denoted by $f_{m^{+}}$, where the subscript $\mathrm{m}^{+}$denotes metal ions (rather than charge-neutral metal atoms). If the mass density of the metal film on the surface is denoted by $\rho$, then the sputtering (i.e., etch) rate due to the metal-ion bombardment becomes

$$
C_{m^{+}}=\frac{m}{\rho} \int_{\mathbf{N} \cdot \mathbf{v}<0} Y_{m^{+}}(\Theta, \nearrow)(\mathbf{N} \cdot \mathbf{v}) f_{m^{+}}(\mathbf{v}, \mathbf{X}) d \mathbf{v} .
$$

Since the incoming ions are the same species as those that form the metal film, the metal ions are simultaneously deposited with the deposition rate

$$
D_{m^{+}}=\frac{m}{\rho} \int_{\mathbf{N} \cdot \mathbf{v}<0}(\mathbf{N} \cdot \mathbf{v}) f_{m}+(\mathbf{v}, \mathbf{X}) d \mathbf{v} .
$$

The normal speed of the surface at position $\mathbf{X}$ due to the metal-ion bombardment is therefore given by $C_{m^{+}}-D_{m^{+}}$, where the positive sign of the speed is taken in the direction of etching, as before.

Likewise the etch rate $C_{g}$ of the metal film due to the inert ion bombardment may be given by

$$
C_{g^{+}}=\frac{m}{\rho} \int_{\mathbf{N} \cdot \mathbf{v}<0} Y_{g^{+}}(\Theta, C)(\mathbf{N} \cdot \mathbf{v}) f_{g^{+}}(\mathbf{v}, \mathbf{X}) d \mathbf{v},
$$

where $f_{g^{*}}(\mathbf{v}, \mathbf{X})$ is the velocity distribution function for the inert-gas ions.

We now assume that the sticking coefficient (probability) of metal atoms-i.e., the probability that a single neutral metal atom impinging on the surface sticks to the surface and stay on the surface-is constant and denoted by .\%. It then follows from Eqs. (2), (4), and (5) that

$$
\nearrow_{m}^{\text {out }}(\mathbf{X})=\left(1-\nearrow^{\prime \text { in }}+\frac{\rho}{m} C_{m^{+}}+\frac{\rho}{m} C_{g^{+}}\right.
$$

i.e., the outgoing flux is the sum of the reemitted metal atoms and the sputtered metal atoms. Here the subscript $m$ for $F_{m}$ out and $\mathcal{F}_{m}^{\text {in }}$ denotes the charge-neutral metal atoms. The equation above gives a relation between the incoming flux $F_{m}$ in and outgoing flux. $F_{m}^{\text {out }}$ of the metal atoms.

The other relation between $T_{m}^{\text {in }}$ and $T^{\text {out }}$ arises from the fact that part of the metal atoms in the gas phase comes from the reemission (if $S<1$ ), and sputtering (if $Y_{m^{+}}$and $Y_{\mathrm{g} m}{ }^{+}$ are nonzero) from the deposited film. ${ }^{7-10}$ We denote the reemission incoming flux-i.e., the metal-atom flux that originates from the surface reemission of the deposited metal film-by $\mathscr{T}^{R}$. Likewise we denote the direct incoming flux 


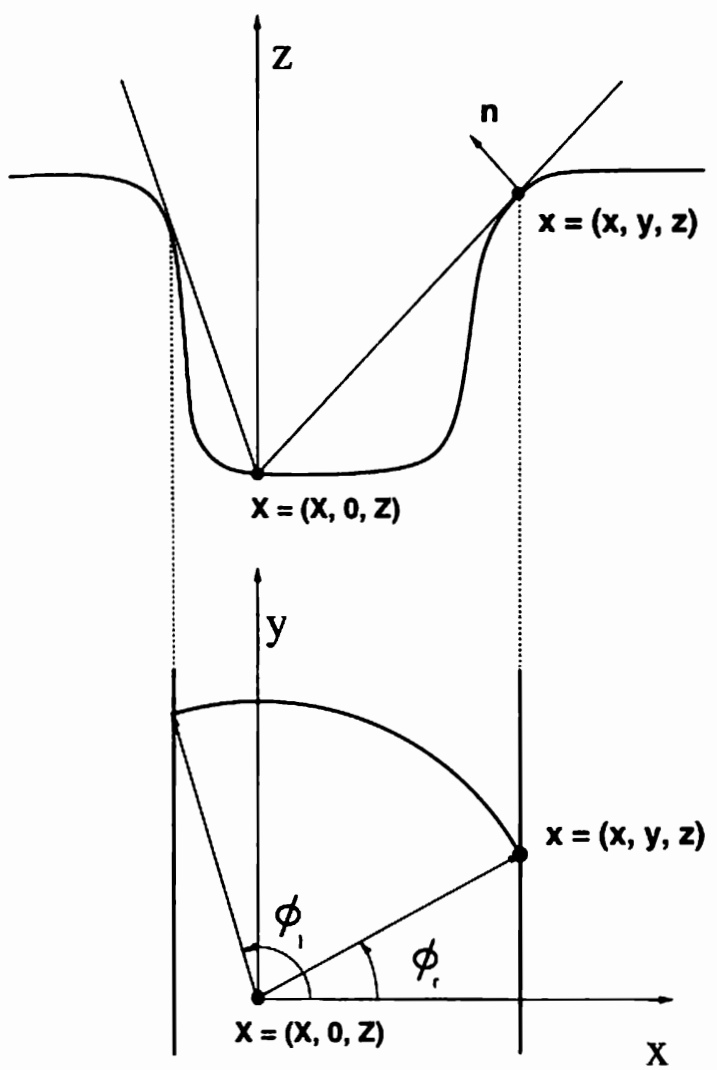

FIG. 2. The side and top views of the trench structure.

from the magnetron cathode-i.e., the metal-atom flux that arrives at the sample surface without having been adsorbed before-by $\mathscr{T}^{D}$. Evidently

$$
T_{m}^{i n}=.^{R}+T^{D}
$$

Since the mean free paths of gas-phase ions and atoms are sufficiently larger than the dimensions of trenches, the transport of the gas-phase particles may be described by the collisionless kinetic equation (i.e., Vlasov equation) and called the Knudsen transport. For example, in the absence of external forces, the transport equation of the metal atoms is given by

$$
\mathbf{v} \cdot \Gamma f_{m}(\mathbf{v}, \mathbf{x})=0
$$

which simply states that the distribution function $f_{m}$ is constant along a straight particle path in the direction of the velocity vector $\mathbf{v}$.

Let the coordinates of point $\mathbf{X}$ on the surface be $(X, 0, Z)$, where we have chosen the origin of the coordinate system in such a way that $\mathbf{X}$ is on the $x-z$ plane. We also choose another point $\mathbf{x}=(x, y, z)$ on the surface, where the unit normal vector of the surface is given by $\mathbf{n}$ (see Fig. 2). If the line segment connecting $\mathbf{X}$ and $\mathbf{X}$ does not intersect any part of the surface except at those end points (i.e., if $\mathbf{x}$ lies on a line of sight from $\mathbf{X}$ ), it follows from Eq. (8) that

$$
f_{m}(\mathbf{v}, \mathbf{X})=f_{m}(\mathbf{v}, \mathbf{x})
$$

for all $v$. Note that $f_{m}(\mathbf{v}, \mathbf{x})$ in Eq. (9) represents the distribution function of the reemitted metal atoms at $\mathbf{x}$ if $\mathbf{v}$ satisfies $\mathbf{n} \cdot \mathbf{v}>0$.

Consider a velocity vector $\mathbf{v}$ that satisfies $\mathbf{n} \cdot \mathbf{v}>0$, i.e., points from $x$ to $X$. Denoting $\mathbf{r}=\mathbf{x}-\mathbf{X}$ and $\hat{\mathbf{r}}=\mathbf{r} /|\mathbf{r}|$, we may write $\mathbf{v}=-v \hat{\mathbf{r}}$. Then the position vector $\mathbf{x}$ becomes a function of $\mathbf{v}$ for fixed $\mathbf{X}$. Using the usual polar coordinate system around the $z$ axis, we may relate $\mathbf{x}$ to $\mathbf{v}$ as

$$
\cos \theta=\frac{\tilde{z}}{r}, \quad \tan \phi=\frac{y}{\tilde{x}},
$$

where

$$
\tilde{x}=x-X, \quad \tilde{z}=z-Z, \quad r=\sqrt{\tilde{x}^{2}+y^{2}+\tilde{z}^{2}} .
$$

Note that the vector $\mathbf{r}=(\tilde{x}, y, \tilde{z})=r \hat{\mathbf{r}}$ is antiparallel to $\mathbf{v}$ $=-v \hat{\mathbf{r}}$.

Therefore, from Eqs. (2) and (9), we obtain

$$
\begin{aligned}
\mathscr{T}^{R} & =-\int_{\mathbf{N} \cdot \mathbf{v}<0}(\mathbf{N} \cdot \mathbf{v}) f^{R}(\mathbf{v}, \mathbf{X}) d \mathbf{v} \\
& =\int R d s \int_{-\infty}^{\infty} d y \int_{0}^{\infty} d v g(\mathbf{X}, \mathbf{x}) v^{3} f^{R}(\mathbf{v}, \mathbf{x}) \frac{(\hat{\mathbf{r}} \cdot \mathbf{N})|\hat{\mathbf{r}} \cdot \hat{\mathbf{n}}|}{r^{2}},
\end{aligned}
$$

where $f^{R}$ denotes the velocity distribution function for the metal ions that are reemitted or sputtered from the deposited film. Note that $f^{R}$ also satisfies Eq. (9). In Eq. (10), the integration over the velocity space was transformed to the integration over $\mathbf{x}$ : $\mathscr{\cap}$ denotes the surface boundary at the $x-z$ plane, $d s=\sqrt{d x^{2}+d z^{2}}$ is the line element on the boundary curve, and therefore $\int_{f_{1}} d s \int_{-x}^{x} d y$ represents the integral over the entire surface. In deriving Eq. (10), we have also used the relations ${ }^{7}$

$$
d \mathbf{v}=v^{2} \sin \theta d v d \theta d \phi=\frac{v^{2}}{r^{2}}|\hat{\mathbf{r}} \cdot \hat{\mathbf{n}}| d s d y d v .
$$

The function $g(\mathbf{X}, \mathbf{x})$ in Eq. (10) is the visibility factor defined by

$$
g(X, X)=\left\{\begin{array}{ll}
1 & \text { if } \mathbf{x} \text { is visible from } \mathbf{X} \\
0 & \text { otherwise }
\end{array} .\right.
$$

For two-dimensional structures, it is evident that we may write

$$
g(\mathbf{X}, \mathbf{X})=g(\mathbf{X}, \boldsymbol{\xi})
$$

with $\boldsymbol{\xi}=(x, 0, z)$, i.e., the projection of $\mathbf{x}$ to the $x-z$ surface.

We further assume that the distribution function $f^{R}(\mathbf{v}, \mathbf{x})$ for the reemitted metal atoms is given by the "generalized" cosine law, i.e.,

$$
f^{R}(\mathbf{v}, \mathbf{x})=f_{v}(v, \boldsymbol{\xi}) \cos ^{\prime \prime} \Theta \text { for } \mathbf{v} \cdot \hat{\mathbf{n}} \geqslant 0,
$$

where $\boldsymbol{\xi}=(x, 0, z), \Theta$ is the angle between vectors $\mathbf{v}$ and $\hat{\mathbf{n}}$ as before, and $\nu(>-1)$ is a parameter. Note that the distribution function $f(\mathbf{v}, \mathbf{x})$ is assumed to be independent of $y$ due to the translational symmetry of the system in the $y$ direction. Then the total outgoing flux of metal atoms from the unit area at $\mathbf{x}$ may be given by 


$$
T_{m}^{\text {out }}(\boldsymbol{\xi})=\int_{0}^{\infty} d v \int_{(v \cdot \hat{\mathbf{n}})>0)} d \Omega v^{3} f_{v}(v, \boldsymbol{\xi}) \cos ^{p+1} \Theta
$$

where $d \Omega$ denotes the solid angle element. From the $\Theta$ dependence of the integrand of Eq. (13), the reemission distribution is called cosine when $\nu=0$. Likewise, an overcosine distribution may be obtained by choosing $\nu>0$ and an undercosine distribution by $-1<\nu<0$.

Substituting Eq. (12) into Eq. (10) and using the relation derived from Eq. (13), i.e.

$$
\int_{0}^{x} v^{3} f_{v}(v, \boldsymbol{\xi}) d v=\frac{(\nu+2)}{2 \pi} \cdot \mathcal{T}_{m}^{\text {out }}(\boldsymbol{\xi}),
$$

we obtain

$$
\begin{aligned}
& T^{R}(\mathbf{X}) \\
& =\frac{(\nu+2)}{2 \pi} \int_{j i} d s \int_{-\infty}^{x} d y g(\mathbf{X}, \boldsymbol{\xi}) \cdot T_{m}^{\text {out }}(\boldsymbol{\xi}) \frac{(\hat{\mathbf{r}} \cdot \hat{\mathbf{N}})|\hat{\mathbf{r}} \cdot \hat{\mathbf{n}}|^{\nu+1}}{r^{2}},
\end{aligned}
$$

where $\cos \Theta=|\hat{\mathbf{r}} \cdot \hat{\mathbf{n}}|$ is used. Carrying out the integration in $y$ then yields

$$
\begin{aligned}
& T^{R}(\mathbf{X}) \\
& \quad=\frac{A_{\nu+4}(\nu+2)}{\pi} \int_{j, j} d s g(\mathbf{X}, \boldsymbol{\xi}) \mathcal{T}_{m}^{\text {out }}(\boldsymbol{\xi}) \frac{(\hat{\mathbf{R}} \cdot \hat{\mathbf{N}})|\hat{\mathbf{R}} \cdot \hat{\mathbf{n}}|^{\nu+1}}{R} .
\end{aligned}
$$

Here $R=|\mathbf{R}|, \hat{\mathbf{R}}=\mathbf{R} / R$, and

$$
\begin{aligned}
A_{\nu+4} & =\int_{0}^{\infty} \frac{d t}{\left(t^{2}+1\right)^{(2+\nu / 2)}} \\
& =\Gamma\left(\frac{\nu+3}{2}\right) \Gamma\left(\frac{1}{2}\right) / 2 \Gamma\left(\frac{\nu}{2}+2\right) .
\end{aligned}
$$

For the cosine distribution $(\nu=0)$, we have $A_{4}=\pi / 4$.

Substituting Eqs. (6) and (7) into Eq. (15), we obtain

$$
\begin{aligned}
& \mathcal{T}^{R}(\mathbf{X})=\frac{A_{\nu+4}(\nu+2)}{\pi}\left((1-. /) \int_{n} d s . T^{R} K_{l \prime}(\mathbf{X}, \boldsymbol{\xi})\right. \\
& +.(\mathbf{X})) \text {, }
\end{aligned}
$$

where the inhomogeneous term $:$ is given by

$$
\begin{aligned}
:(\mathbf{X})= & \int_{ת} d s K_{l}(\mathbf{X}, \boldsymbol{\xi})\left((1-. /) \cdot T^{D}(\boldsymbol{\xi})\right. \\
& \left.+\frac{\rho}{m}\left[C_{m^{+}}(\boldsymbol{\xi})+C_{g^{+}}(\boldsymbol{\xi})\right]\right)
\end{aligned}
$$

and $K_{\nu}(\mathbf{X}, \boldsymbol{\xi})$ is the kernel defined by

$$
K_{\nu}(\mathbf{X}, \boldsymbol{\xi})=g(\mathbf{X}, \boldsymbol{\xi}) \frac{(\hat{\mathbf{R}} \cdot \hat{\mathbf{N}})|\hat{\mathbf{R}} \cdot \hat{\mathbf{n}}|^{\nu+1}}{R} .
$$

The flux $\zeta(\mathbf{X})$ may be calculated from the knowledge of the distribution functions for metal ions, inert-gas ions, and the metal atoms that are directly emitted from the magnetron target. The flux.$\tau^{R}$ of the reemitted metal atoms is, on the other hand, obtained from the integral equation (16) once $\because(\mathbf{X})$ is given.

Writing $D^{R}=(m . / / \rho) \cdot T^{R}$ and $D^{D}=(m . / / \rho) \cdot T^{D}$, we obtain the rate of metal deposition as

$$
D=D^{R}+D^{D}+D_{m^{+}},
$$

i.e., the sum of the deposition rates due to the metal atoms reemitted from the surface, the metal atoms directly from the magnetron cathode target, and the metal ions generated in the plasma. Since the deposited film is simultaneously etched by metal and inert gas ions, the net normal velocity of the surface at $\mathbf{X}$ is given by $C-D$ with $C=C_{m^{+}}+C_{g}+$. Here the positive sign of the normal velocity is taken in the direction of etching as before.

The neutral metal flux emitted directly from the magnetron cathode target may be given at $\mathbf{X}$ on the sample surface by

$$
T^{-1)}(\mathbf{X})=\int_{\mathbf{N} \cdot \mathbf{v}<0}(\mathbf{N} \cdot \mathbf{v}) g(\mathbf{X}, \mathbf{x}) f^{l)}(\mathbf{v}, \mathbf{x}) d \mathbf{v},
$$

where $f^{D}(\mathbf{v}, \mathbf{x})$ is the distribution function of the sputtered metal ions at $\mathbf{x}$ on the target cathode surface. Note that we have used Eq. (8) to replace the distribution function on the sample surface by $f^{D}(\mathbf{v}, \mathbf{x})$. [Note also that some of the metal atoms sputtered from the target become ionized in the plasma, and thus do not arrive at $\mathbf{X}$ as charge-neutral particles. In Eq. (19), therefore, we consider $f^{D}(\mathbf{v}, \mathbf{x})$ as the distribution function only for those metal atoms that reach $\mathbf{X}$ without being ionized.]

It is now assumed that the distribution function $f^{l)}$ on the target is isotropic-i.e., independent of the direction of $\mathbf{v}$-and independent of position $\mathbf{x}: f^{D}(\mathbf{v}, \mathbf{x})=f^{D}(v)$. This distribution is often referred to as the "cosine" distribution of the sputtered flux since the cosine function dependence arises from the inner product $(\mathbf{N} \cdot \mathbf{v})$ in Eq. (19). Note that the distribution function $f^{D}$ on the target generally differs from the reemission distribution function $f^{R}$ [Eq. (12)], for which we have assumed the generalized cosine distribution with the adjustable parameter $\nu$.

Choosing the usual polar angles $\theta$ and $\phi$ around the : direction again, it is straightforward to evaluate Eq. (19):

$T^{D}=\frac{1}{\pi} \int_{0}^{\theta_{\max }} d \theta \int_{\phi_{r}(\theta)}^{\phi_{I}(\theta)}\left(N_{x} \sin \theta \cos \phi+N_{z} \cos \theta\right) G(\theta)$.

where $\mathbf{N}=\left(N_{x}, 0, N_{-}\right)$and $G(\theta)$ denotes the differential flux

$$
G(\theta)=2 \pi \sin \theta \int_{0}^{\infty} d v f^{D}(v) .
$$

In Eq. (20), $\theta_{\max }$ is the maximum angle ${ }^{12}$ that $\theta$ can take. which usually less than $\pi / 2$ due to the flux collimation (see Sec. III). The limits of the azimuthal angle $\phi_{r}(\theta)$ and $\phi_{l}(\theta)$. which are functions of $\theta$, arise from the two-dimensional nature of the structure [see Fig. 2(b)]. The integration over $\phi$ may be easily carried out. 


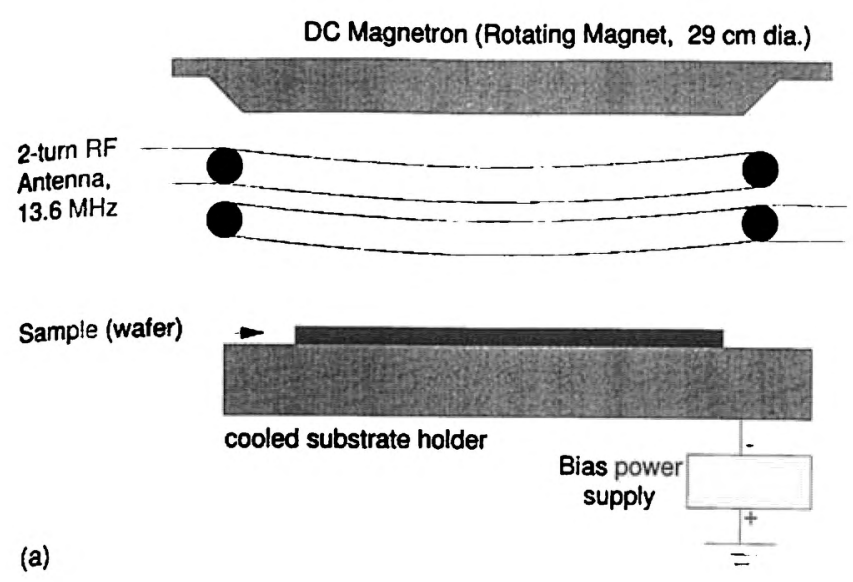

target

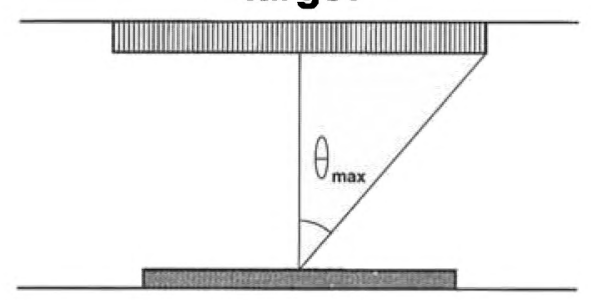

(b)

\section{wafer}

FIG. 3. (a) Experimental apparatus. (b) The collimation angle $\theta_{\max }$ due to the finite target.

In particular, if the sample is completely flat, then we have $\mathbf{N}=(0,0,1), \phi_{r}(\theta)=0$, and $\phi_{l}(\theta)=\pi$. Therefore, this flatsurface flux of the metal atoms becomes

$$
. / /\left(\theta_{\max }\right)=\frac{\pi}{2}\left(1-\cos \theta_{\max }\right) \int_{0}^{v} d v v^{3} f^{D}(v)
$$

Using this expression, Eq. (20) may be recast as

$$
\begin{aligned}
\not \mathscr{F}^{D}(\mathbf{X})= & \frac{4 . / /\left(\theta_{\max }\right)}{\pi\left(1-\cos 2 \theta_{\max }\right)} \int_{0}^{\theta_{\max }} d \theta \int_{\phi_{r}(\theta)}^{\phi_{l}(\theta)}\left(N_{x} \sin ^{2} \theta \cos \phi\right. \\
& \left.+N_{z} \sin \theta \cos \theta\right) d \phi .
\end{aligned}
$$

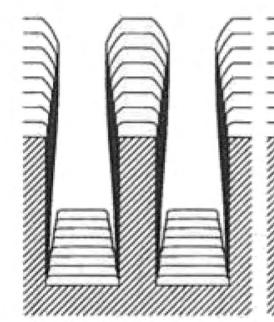

(a)

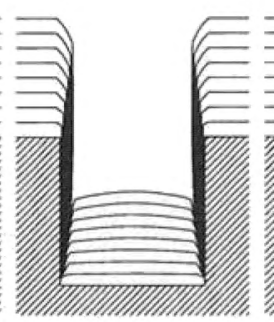

(b)

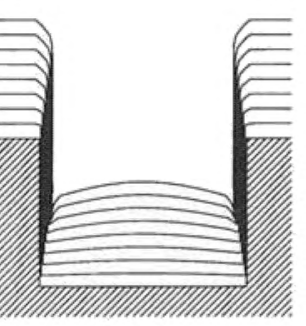

(c)
FIG. 4. Simulations of $\mathrm{Al}$ deposition into trenches with various aspect ratios: (a) 2.0, (b) 1.0, and (c) 0.714 . The ion-to-neutral flux ratio is $1: I$ and the sputtering yield is 0 . Also the sticking coefficient $\searrow=1$ and the cosine resputter distribution (i.e., $\nu=0$ ) is assumed.

Due to the dc-bias voltage applied to the wafer, ions impinge on the wafer surface vertically if collisions in the sheath are negligibly small. Therefore we assume for simplicity that the ion fluxes are unidirectional and independent of position $\mathbf{X}$. Then the metal-ion distribution functions may be given as

$$
f_{m}+(\mathbf{v})=f_{m}+(v) \frac{\delta(\theta)}{\sin \theta} .
$$

The distribution function $f_{g}+(\mathbf{v})$ for the inert-gas ions is also given similarly.

Substituting Eq. (22) into Eq. (4), we obtain the etchrate $C_{m}+(\Theta)$ due to the metal ions as a function of the angle $\Theta$ formed by the surface normal $\mathbf{N}$ and the $z$ axis (i.e., $N_{z}=\cos \Theta$ ). The angle $\Theta$ also represents the slope angle measured from the $x$ axis (see Fig. 1). In particular, on the flat surface, the total flux of metal ions and the corresponding etch rate are given by

$$
\mathbb{H}^{+}=\int_{0}^{\infty} d v v^{3} f_{m}+(v)
$$

and

$$
C_{m}+(0)=\frac{2 \pi m}{\rho} \int_{0}^{\infty} d v v^{3} Y_{m^{+}}+\left(0, m v^{2} / 2\right) f_{m^{+}}+(v)
$$

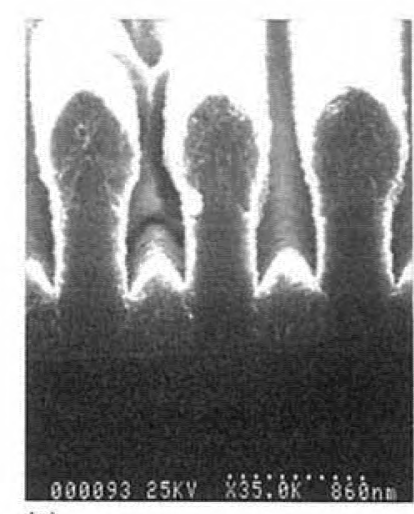

(a)

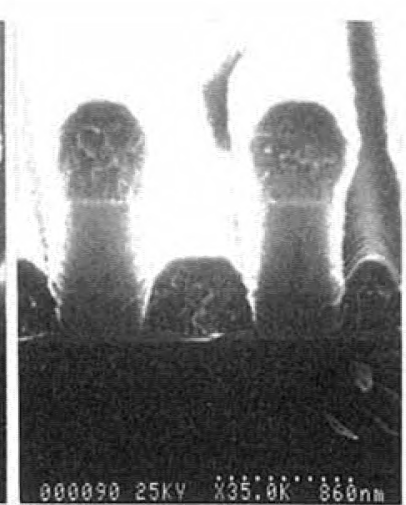

(b)

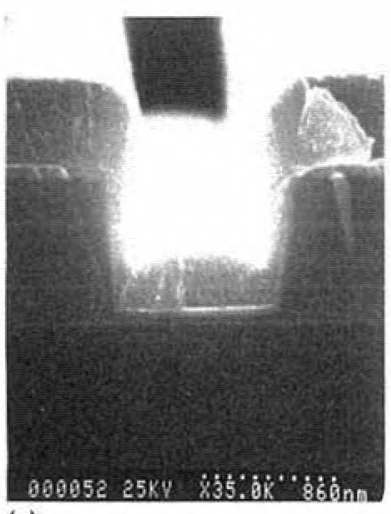

(c)

FIc. 5. SEM pictures of Al deposition under the conditions similar to Fig. 4. 


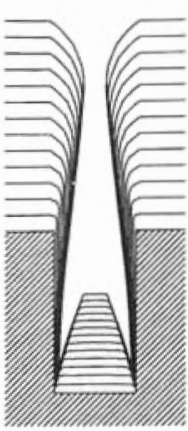

(a)

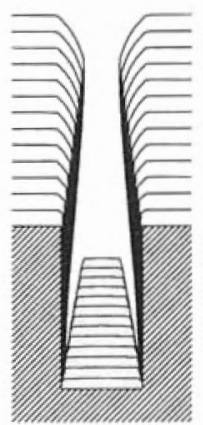

(b)

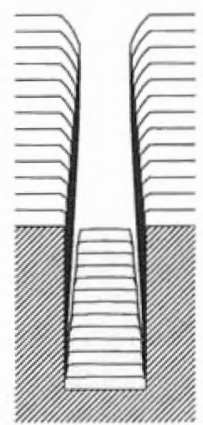

(c)
FIG. 6. Simulations of Al deposition with various ion-10-neutral flux ratios: (a) 1:2, (b) $1: 1$, and (c) 2:1. The other conditions are the same as those used in Fig. 4.

For a general angle $\Theta$, we thus obtain

$$
C_{m}+(\Theta)=C_{m}+(0) \not / m+(\Theta) \cos \Theta,
$$

where we have assumed that $Y_{m^{+}}(\Theta, \mathscr{C})$ $=1 / m^{+}(\Theta) Y_{m}+(0, \circlearrowright)$ for simplicity. [Note that $1 / m^{+}+(0)$ =1.] Similarly the etchrate $C_{g}+$ of the deposited metal film due to the inter gas ions may be expressed as

$$
C_{g}+(\Theta)=C_{g}+(0) / / g^{+}(\Theta) \cos \Theta .
$$

The expressions given in Eqs. (21), (24), and (25) are used to evaluate the inhomogeneous term in Eq. (17).

\section{EXPERIMENTAL SETUP}

Figure 3(a) shows a schematic diagram of the ionized magnetron sputtering system. Details of the experiments may be found in Refs. 1 and 2. Without turning on the two-turn RFI coil, the system is a conventional dc magnetron sputter tool with a rotating magnet. The magnetron power may be varied up to $10 \mathrm{~kW}$ on the $200 \mathrm{~mm}$ target cathode (Al for the experiments presented in this article) and up to $30 \mathrm{~kW}$ on the $300 \mathrm{~mm}$ cathode. A Si wafer of diameter $200 \mathrm{~mm}$ is mounted on a holder $90 \mathrm{~mm}$ below the magnetron cathode (target). The sputtered area on the cathode under the magnetron operation is of diameter $230 \mathrm{~mm}$, so the collimation angle $\theta_{\max }$ shown in Fig. 3(b) is given by $\tan \theta_{\max }=115 / 90$, i.e., $\theta_{\max }=0.907=52.0^{\circ}$. Plasmas are generated by applying $13.56 \mathrm{MHz}$ power (up to $3 \mathrm{~kW}$ ) to the RFI coil. In our experiments, the dc-bias voltage on the substrate was varied up to $-200 \mathrm{~V}$ and the Ar pressure was varied from 1 to 35 mTorr.

When higher ionization of the metal atoms is desirable, the pressure is maintained relatively high (about $35 \mathrm{mTorr}$ ). For example, at pressure $35 \mathrm{mTorr}$, the minimum mean free path is about $2.5 \mathrm{~mm}$, which is derived from the peak value of the charge-exchange cross section (as a function of the kinetic energy) $40 \AA^{2}$. In this case, virtually all of the sputtered atoms are slowed down by collisions in the region between the target and sample and thus the rate of ionization increases. Note, however, that collisions in the sheath region
( $\approx 0.1 \mathrm{~mm}$ thick) at the sample wafer are negligible, so that the ions impinging on the sample surface may be assumed unidirectional.

Under low pressure conditions (e.g., in the traditional magnetron sputtering mode), the mean free paths become comparable to or larger than the tool dimension. Then, even without collimators, "the finite size of the target effectively limits the angular distribution of the metal atoms impinging on the sample surface. In this article, we only consider microscopic structures located at the center of the wafer [Fig. $3(b)]$. Then the limit angle of the metal atom distribution function is given by the polar angle $\theta_{\max }=52.0^{\circ}$. The effects of collimators, which would typically be placed about 50 $\mathrm{mm}$ below the magnetron cathode and thus further limit the angular distribution of the metal atoms, will be discussed in a future publication.

The trench structures that are to be filled with the metal in the experiments are typically less than $1 \mu \mathrm{m}$ deep and have various trench widths and sidewall angles. The aspect ratio of the trench is defined as the ratio of the depth to width, as

(a)
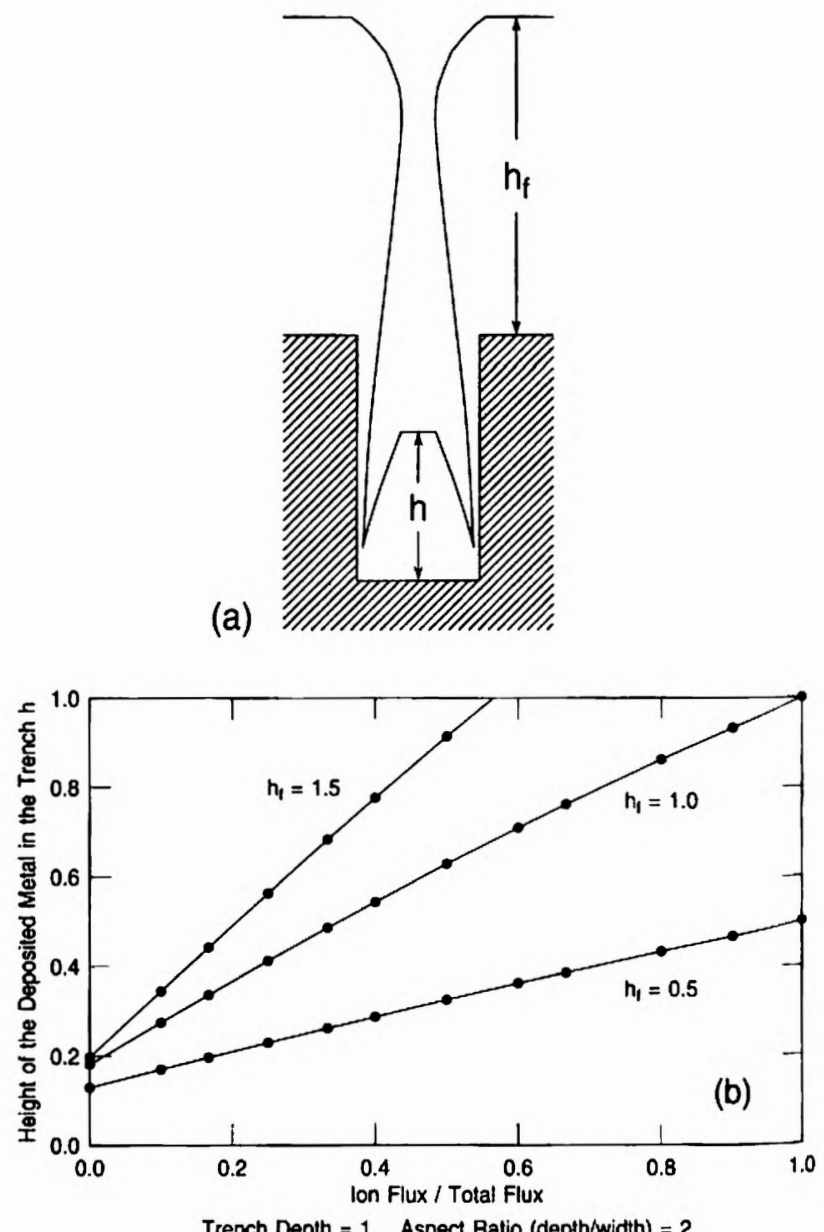

FIG. 7. The relation between the film thickness in the trench and the ion-10neutral flux ratio. (a) Definitions of the film thicknesses on the top surface $h_{f}$ and in the trench $h$. (b) The relation between $h$ and the ratio $R$ of the ion flux to the total flux (i.e., the sum of the ion and neutral fluxes) for various $h_{f}$, obtained from the simulations. 


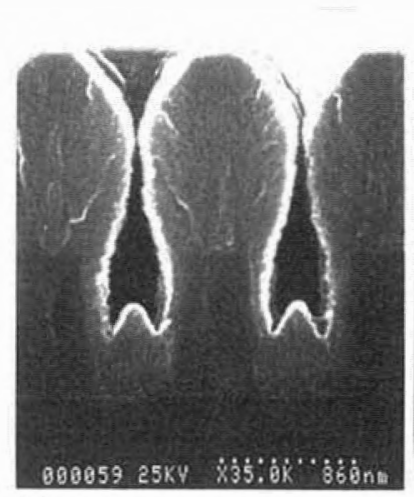

(a)

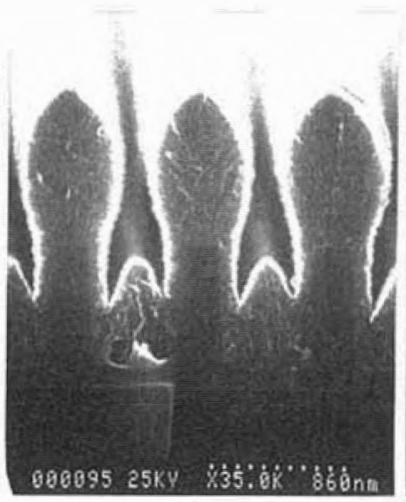

(b)

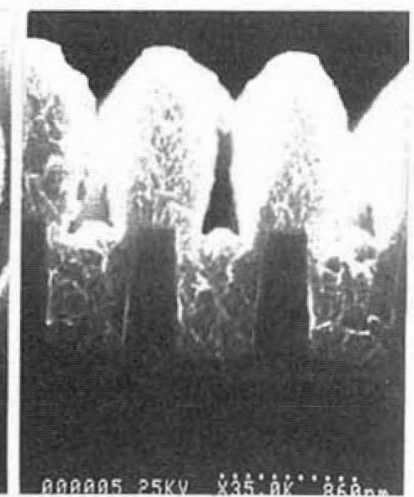

(c)

FIG. 8. SEM pictures of Al deposition under the conditions similar to Fig. 6. The ion-to-neutral flux ratios are approximatcly (a) 3:7, (b) 1:1, and (c) 7:3.

usual. The trenches are made of etched $\mathrm{SiO}_{2}$ on $\mathrm{Si}$ and the wafer temperature is usually maintained at less than $100^{\circ} \mathrm{C}$ during the ionized sputter deposition process.

\section{NUMERICAL AND EXPERIMENTAL RESULTS}

In this section, we shall present some sample calculations and compare the numerical results to experimental observations. In the light of the relatively simple physical model that we have employed (i.e., the unidirectional ion flux, uniform angular distribution of neutral flux with the collimation cutoff $\theta_{\text {mix }}$, no surface diffusion, etc.), however, we do not attempt to reproduce exact profiles that experiments exhibit by our simulations. The goal here is to demonstrate the validity of our physical model, and that the simulations can indeed predict correct tendency of experimental observations as one varies experimental conditions.

Figure 4 shows simulation results of the metal deposition into trenches with three different aspect ratios (i.e., 2, 1, and 0.7141 . The shaded area represents the initial $\mathrm{SiO}_{2}$ trenches. The sticking coefficient $. Y=1$ and sputtering yields $Y_{m+}(\omega)=Y_{g}+(\theta)=0$ (and therefore no etching taking placel are assumed. The ratio of the flat-surface ion flux to the flat-surface neutral fluxes (after collimation) is assumed to be $. / 1,+: / /\left(\theta_{\max }\right)=1: 1$. Each solid line in the figures represents the profile of deposited metal at equal time intervals.

The collimation angle $\theta_{\max }=0.907=52.0^{\circ}$ is employed for all the simulations presented in this article. Strictly speaking, this is valid only for low-pressure discharges. For relatively

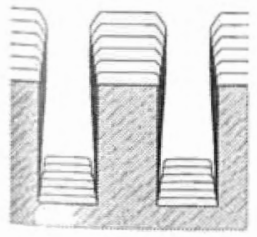

(a)

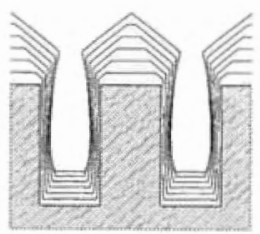

(b)

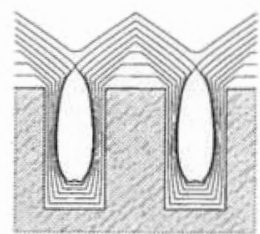

(c)
FIG. 9. Simulations of $\mathrm{Al}$ deposition with various total sputtering yields: (a) 0 . (b) 0.6, and (c) 0.8. The other conditions are the same as those used in Fig. 4 . high-pressure discharges (e.g., $35 \mathrm{mT}$ Torr), sputtered atoms at the magnetron target are sufficiently randomized through collisions and the cutoff angle $\theta_{\max }$ is expected to be close to $90^{\circ}$. Our simulations show, however, that the uniform angular distribution of the neutral $\mathrm{Al}$ atoms with $\theta_{\max }=90^{\circ} \mathrm{sig}$ nificantly overestimates the contribution from the lateral component of the neutral flux when compared with experiments. This may be attributed to the nonisotropic angular distribution $f^{D}(\mathbf{v}, \mathbf{x})$. Since the true distribution function $f^{D}$ is not readily available from the experiments, we employ the isotropic distribution with $\theta_{\max }=52^{\circ}$ even for high-pressure discharges up to $35 \mathrm{~m}$ Torr. So the simulation results presented here somewhat underestimate the contribution from the neutral-flux lateral component in high-pressure discharges and thus provide slightly more optimistic results for trench filling.

Figure 5 shows the SEM pictures of $\mathrm{Al}$ deposition into trench structures obtained under conditions similar to Fig. 4. The dc magnetron cathode and RFI coil were powered at 2 and $1 \mathrm{~kW}$, and Ar pressure $35 \mathrm{mTorr}$ was used. The dc-bias voltage at the wafer was $20 \mathrm{~V}$, so that the sputtering due to the $\mathrm{Al}$ and $\mathrm{Ar}$ ions was negligible. The ratio of the ion flux to neutral flux is approximately $1: 1$ under these conditions.

In Fig. 6, numerical results of trench filling with aspect ratio 2 are shown for different flat-surface flux ratios. The conditions used in these calculations are the same as those in Fig. 4(a) except that the ion-to-neutral flux ratios $. \mathbb{H}^{+}: . \mathscr{L}\left(\theta_{\max }\right)=2: 1,1: 1$, and $1: 2$ are used for Figs. 6(a), $6(\mathrm{~b})$, and $6(\mathrm{c})$, respectively. The total incoming flux $. / I^{+}+. / 1\left(\theta_{\max }\right)$ is held constant for Figs. $6(a)-6(c)$, and therefore the thicknesses of the deposited film on the top $\mathrm{Si}$ surface [which is denoted by $h_{f}$ in Fig. $7(a)$ ] are the same among these figures.

Evidently, under the conditions used in Fig. 6, the height of the deposited metal in the trench, which is denoted by $h$ in Fig. 7(a), is a function of the ratio of the ion flux to the total flux $R=. \mathbb{Z}^{+} /\left[. \mathbb{Z}^{+}+. / /\left(\theta_{\max }\right)\right]$ and the height of the top surface film $h_{f}$. This information may be used to estimate the ion-to-neutral flux ratio if its direct measurement at the sample surface is not readily available. For example, our numerical simulations predict that $h$ depends on $R$ as shown 


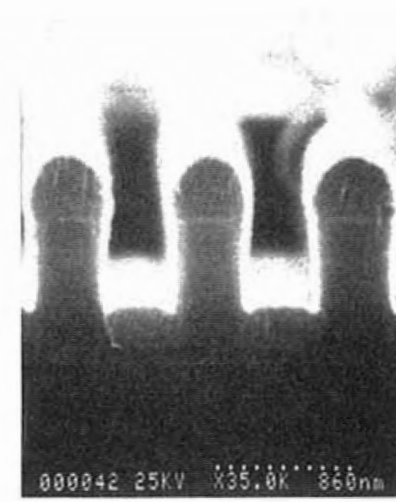

(a)

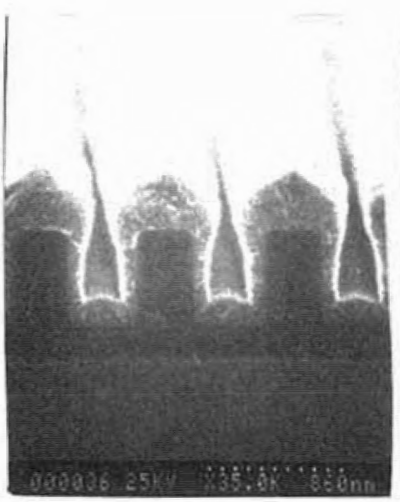

(b)

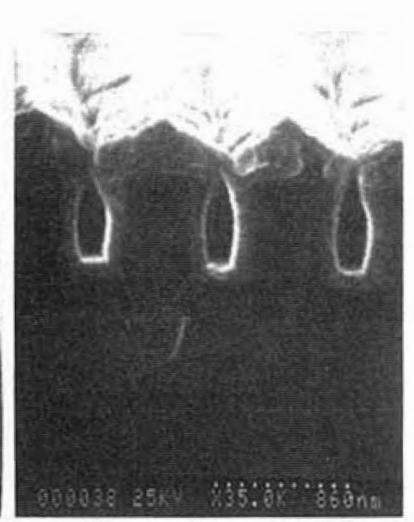

(c)

FIG. 10. SEM pictures of Al deposition under the conditions similar to Fig. 6 . The ion energies at the sample surface are approximately (a) 20 eV or less. (b) $80 \mathrm{eV}$, and (c) $120 \mathrm{eV}$ or more.

in Fig. 7(b), for different values for $h_{f}$ when the trench depth is 1 and aspect ratio is 2 . Indeed, lengths are normalized by the trench depth in these simulations. Therefore the value of $h_{f}($ or $h)$ in Fig. 7(b) may be interpreted as the ratio of the film thickness on the top surface (or in the trench) to the trench depth when compared with experiments. Experimental determination of the relation between $h$ and $R$ under various conditions, including verification of Fig. 7(b), will be the subject of future studies. For a small flux ratio $R$, a void may be formed when $h_{f}$ becomes sufficiently large and thus $h$ may have the maximum value (as a function of $R$ ). For example, the simulations show that the maximum value of $h$ is 0.20 for $R=0$.

Figure 8 shows SEM pictures of the deposition cross sections for trench filling as a function of increasing ion-toneutral flux ratio, similar to the simulations shown in Figs. 6(a)-6(c). The ion-to-neutral flux ratios were directly measured in the experiments. ${ }^{2}$ Figure $8(a)$ is for the case of the lowest relative ionization: the ion-to-neutral flux ratio is approximately $3: 7$. Figures $8(\mathrm{~b})$ and $8(\mathrm{c})$ show the effect of increasing the relative ionization to approximately $1: 1$ and $7: 3$, respectively. In these runs, the magnetron discharge power, and hence the flux of neutral metal into the discharge was kept constant, and the rf power to the RFI coil increased. The relative magnetron-to-RFI power ratios were about 3:1 for Fig. 8(a), 2:1 for Fig. 8(b), and 1:1 for Fig. 8(c).

Figure 9 shows the effects of increased sputtering at the sample surface, which may be compared with experimental results shown in Fig. 10. In these simulations, the ion-toneutral flux ratio is $1: 1$ and the total sputtering yield $Y$ $=Y_{m^{+}}+Y_{g^{+}}$at the slope angle $\Theta=0$ is 0 for Fig. 9(a), 0.6 for Fig. 9(b), and 0.8 for Fig. 9(c). Note that the total sputtering yield $Y$, rather than individual $Y_{m^{+}}$and $Y_{g^{+}}$determines the deposition profile in our model if both $\mathrm{Al}$ and $\mathrm{Ar}$ ions have the same velocity distribution function at the sample surface (e.g., unidirectional in our examples) and the sputtering yields $Y_{m}+(\Theta, C)$ and $Y_{g},(\Theta, C)$ for $\mathrm{Al}$ and $\mathrm{Ar}$ may be assumed to have the same functional forms. [See Eqs. (3)-(5).] The dependence of the sputtering yield $Y$ on the slope angle (i.e., ion beam incident angle) $\Theta$ used for the simulations was taken from the experimental data of the $\mathrm{Al}$ etch rate measured by Lee. ${ }^{13}$ Although these data are based on $300 \mathrm{eV}$ Ar beam experiments, rather than low-energy $(\$ 120 \mathrm{eV}) \mathrm{Al}$ and $\mathrm{Ar}$ ions that we used in the experiments, we postulate that the functional form $Y(\Theta)$ derived from Ref. 13 does not essentially differ from the true function. [The absolute value of the etch rate is, however, readjusted in such a way that the total deposition rate on the flat surfaceincluding the sputtering effect-becomes 1 for given $Y(0)$ in Figs. 9(a)-9(c).] In the simulation code, cubic splines are used to represent a fitting curve for the etch rate data given in Ref. 13, as shown in Fig. 11.

It may be conjectured that sputtering at the depositing film surface would be a desirable effect; the sputtering should etch away any overhanging deposits and leave the trench structure wide open. This is indeed what occurs when the trench width is sufficiently larger than the deposited film thickness. However, as the film thickness becomes compa-

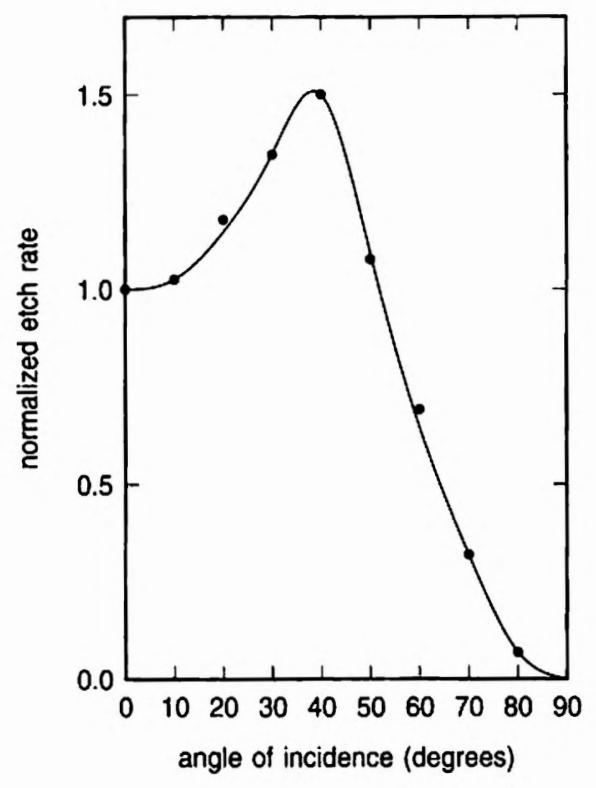

FIG. 11. The dependence of the normalized etch rate [which is proportional to $Y(\Theta) \cos \Theta]$ for $A l$ as a function of the slope angle (i.e., angle of ion beam incidence) $\Theta$. Solid circles are experimental data taken from Ref. 13 and the fitting curve is represented by cubic splines. 
rable with the trench width, the atoms sputtered from one side of the trench are collected on the opposite side. This leads to a lateral buildup of resputtered materials, which can eventually result in closing off the trench [Fig. 9(c)].

The experimental depositions of this same effect are shown in Figs. 10(a)-10(c). The film shown in Fig. 10(a) was deposited with ion energies of $20 \mathrm{eV}$ or less: enough to cause a directional deposition, but insufficient to cause major resputtering at the surface. Figure 10(b) shows the effect of increasing the ion energy to about $80 \mathrm{eV}$, and Fig. 10(c) shows the result with ion energies of $120 \mathrm{eV}$ or more. Although we have not yet determined the relationship between the ion energy and the sputtering yield $Y$, the simulation results clearly show the correct tendency of the deposition profiles with increasing ion energies.

\section{v. CONCLUDING REMARKS}

We have presented numerical calculations of microscopic surface profile evolutions under ionized magnetron sputter metal deposition. The numerical algorithm for the simulations is based on the shock-tracking method, ${ }^{6}$ which solves the equation of moving surfaces with high accuracy.

Under general conditions, the evolving surfaces are subject to simultaneous deposition (due to impinging neutral and ionic metal species) and etching (due to sputtering by metal and incrt-gas ions). The local deposition and etch rates are calculated from the incoming fluxes at the surface. The calculations of the fluxes involve the solution to the integral equation (16), which describes the self-consistent transport of the reemitted metal atoms. [Note however that, if $. Y=1$, Eq. (16) becomes simplified and no longer an integral equation.] Since the dimensions of the microscopic features (i.e., trench structures) are sufficiently smaller than the mean-free paths of the gas-phase species, the transport of ions and neutral atoms is of Knudsen type.

The parameters that need to be specified for the simulation model are the sputtering yield curves $Y_{m}+(\Theta)$ and $Y_{\mathrm{g}^{+}}(\Theta)$ as functions of the slope angle $\Theta$ for metal and inert-gas ions, the sticking coefficient $Y$ for the metal atoms, the degree $\nu$ of the generalized cosine law for sputtered atoms, and the fluxes of inert-gas ions, metal atoms, and metal ions from the ionized magnetron source. The velocity distributions of ions at the sample surface are assumed to be unidirectional and the neutral metal distribution from the plasma/sputtering source is assumed to be isotropic with the collimation angle $\theta_{\max }$. Surface diffusion of metal atoms are assumed to be negligible. In the cases presented here, $\mathscr{Y}=1$ and $\nu=0$ (i.e., usual cosine distribution) are used.

As we have shown in Sec. IV, the simulation model yields correct profiles of deposited metal in trench structures with various aspect ratios. This vindicates our assumptions for the model described in Sec. II. More quantitative comparison between simulation results and experiments, as well as use of the simulations to optimize the profile control, will be presented in future publications. These later results will also show the effects of varying the ion energy and hence the amount of resputtering at the wafer surface in order to control the film profiles.

'S. M. Rossnagel and J. Hopwood, J. Vac. Sci. Technol. B 12, 449 (1994) ${ }^{2}$ S. M. Rossnagel and J. Hopwood, Appl. Phys. Lett. 63, 3285 (1993).

${ }^{3}$ W. Holber, J. S. Logan, H. J. Grabarz, J. T. C. Yeh, J. B. O. Caughman, A. Sugarman, and F. E Turene, J. Vac. Sci. Technol. A 11, 2093 (1993).

${ }^{4} S$. Takehiro, N. Yamanaka, H. Shindo. S. Shingubara, and Y. Horiike, Jpn. J. Appl. Phys. 30, 3657 (1991).

${ }^{5}$ T. Ono, C. Takahashi, and S. Matsuo. Jpn. J. Appl. Phys. 23, L534 (1984)

'S. Hamaguchi, M. Dalvie, R. T. Farouki, and S. Sethuraman, J. Appl. Phys. 74, 5172 (1993).

${ }^{7}$ S. Hamaguchi and M. Dalvie, J Vac. Sci. Technol. A 12, 2745 (1994).

${ }^{8}$ S. Hamaguchi and M. Dalvie. J. Electrochem. Soc. 141, 1964 (1994).

"V. K. Singh, S. G. Shaqfeh, and J. P. McVittie, J. Vac. Sci. Technol. B 12. 2952 (1994).

${ }^{10}$ V. K. Singh, S. G. Shaqfeh. and J. P. McVittie, J. Vac. Sci. Technol. B 10, 1091 (1992).

"S. M. Rossnagel, D. Mikalsea. H. Kinoshita. and J. J. Cuomo. J. Vac. Sci Technol. A 9. 26I (1991).

${ }^{12}$ G. M. Turner, S. M. Rossnagel, and J. J. Cuomo, J. Vac. Sci. Technol. A 11, 2796 (1993)

${ }^{13}$ R. E. Lee, J. Vac. Sci. Technol. 16, 164 (1979). 